\title{
BMJ Global Health Roll-out of HIV pre-exposure prophylaxis: a gateway to mental health promotion
}

\author{
Daniel J Ikeda (D , , ${ }^{1}$ Khameer Kidia, ${ }^{2}$ Bruce D Agins, ${ }^{3}$ Jessica E Haberer, ${ }^{4}$ \\ Alexander C Tsai (D) ${ }^{4}$
}

To cite: Ikeda DJ, Kidia K, Agins BD, et al. Roll-out of HIV pre-exposure prophylaxis: a gateway to mental health promotion. BMJ Global Health 2021;6:e007212. doi:10.1136/ bmjgh-2021-007212

Handling editor Seye Abimbola

Received 18 August 2021 Accepted 25 November 2021

\section{Check for updates}

(c) Author(s) (or their employer(s)) 2021. Re-use permitted under CC BY-NC. No commercial re-use. See rights and permissions. Published by BMJ.

${ }^{1}$ Harvard Medical School, Boston, Massachusetts, USA 2Division of Global Health Equity, Brigham and Women's Hospital, Boston, Massachusetts, USA ${ }^{3}$ Institute for Global Health Sciences, University of California San Francisco, San Francisco, California, USA

${ }^{4}$ Center for Global Health and Mongan Institute,

Massachusetts General Hospital Boston, Massachusetts, USA

Correspondence to

Daniel J Ikeda;

daniel_ikeda@hms.harvard.edu

\section{ABSTRACT}

HIV remains a pressing global health problem, with 1.5 million new infections reported globally in 2020 . HIV preexposure prophylaxis (PrEP) can lower the likelihood of HIV acquisition among populations at elevated risk, yet its global roll-out has been discouragingly slow. Psychosocial factors, such as co-occurring mental illness and substance use, are highly prevalent among populations likely to benefit from PrEP, and have been shown to undermine persistence and adherence. In this analysis, we review the high burden of mental health problems among PrEP candidates and contend that inattention to mental health stands to undermine efforts to implement PrEP on a global scale. We conclude that integration of mental health screening and treatment within PrEP scale-up efforts represents an important strategy for maximising PrEP effectiveness while addressing the high burden of mental illness among at-risk populations. As implementers seek to integrate mental health services within PrEP services, efforts to keep access to PrEP as low-threshold as possible should be maintained. Moreover, programmes should seek to implement mental health interventions that are sensitive to local resource constraints and seek to reduce intersecting stigmas associated with HIV and mental illness.

\section{INTRODUCTION}

HIV remains a significant global health problem. In 2020, an estimated 680000 individuals died from HIV-related causes globally, while there were 1.5 million new infections. ${ }^{1}$ Although HIV-related mortality has declined nearly $50 \%$ over the past decade, declines in new infections have been sluggish, particularly among key populations at elevated risk of infection (eg, men who have sex with men, transgender people, people who inject drugs and adolescent girls and young women). To address the high burden of new infections among these populations, there exists a pressing need to rapidly expand access to high-quality HIV prevention programming.

The introduction of oral pre-exposure prophylaxis (PrEP) was a watershed moment

\section{Summary box}

HIV remains a global health problem, with millions of new infections each year. Pre-exposure prophylaxis (PrEP) can substantially reduce the risk of HIV infection; however, its scale up has been slower than hoped, reaching only a fraction of individuals likely to benefit from its use.

- Psychosocial factors, such as co-occurring mental illness and substance use, are highly prevalent among candidates for PrEP, with some studies reporting prevalence of mental illness as high as $20 \%$.

- Findings from both qualitative and quantitative studies implicate poor mental health as a barrier to PrEP uptake and persistence, suggesting a need to address mental health as part of PrEP implementation.

- Integration of mental health screening and treatment within PrEP scale-up efforts represents an important strategy for maximising PrEP effectiveness while addressing the high burden of mental illness among at-risk populations. The feasibility of this strategy is supported by studies from both high-income and low-income and middle-income countries showing the potential population health benefits of integrating primary care services within existing HIV prevention and treatment programmes.

in the history of HIV prevention. When taken on a daily or on-demand basis, PrEP use can substantially lower the likelihood of HIV acquisition. $^{2}{ }^{3}$ Yet despite PrEP's immense promise, its global scale up has been slow. ${ }^{4}$ Like HIV treatment, high-quality PrEP services can be conceptualised according to a 'cascade' of necessary, sequential steps, extending from PrEP awareness and initiation through to PrEP adherence and retention. ${ }^{5}$ Persistence (ie, ongoing use) and adequate adherence to recommended dosing in the setting of HIV risk are key components of the PrEP cascade that, if not effectively promoted and supported, have the potential to undermine PrEP's effectiveness. ${ }^{6}$ To be maximally protective, PrEP use must consistently coincide with potential HIV exposure 
events (ie, prevention-effective adherence), ${ }^{7}$ but data suggest that PrEP discontinuation is common, with many individuals discontinuing PrEP within the first 6 months of initiation. ${ }^{8}$ Although discontinuation of PrEP in the absence of anticipated HIV exposure may be 'strategic, effective and efficient, ${ }^{7}$ people who discontinue PrEP in the setting of ongoing exposure events and inconsistent or absent condom use during sexual intercourse may be at risk for HIV acquisition. ${ }^{9}$

Psychosocial factors are a central concern in PrEP discontinuation. Empirical data about predictors of PrEP persistence are scant, but a recent qualitative study of people recruited through San Francisco safety-net clinics who acquired HIV despite initiating PrEP implicated substance use and poor mental health as contributors to discontinuation. ${ }^{10}$ Mental disorders have long been shown to amplify the risk of HIV acquisition among men who have sex with men through diminished self-efficacy, increased compulsivity, and reduced engagement in HIV preventive behaviours. ${ }^{11} 12$ High rates of mental disorders have also been reported among other at-risk populations, including adolescent girls and young women in sub-Saharan Africa. ${ }^{13}$ Unfortunately, screening and treatment rates for these disorders remain low, particularly in low-income and middle-income countries (LMICs) with high burdens of HIV. Given the mutually causal link between mental disorders and HIV risk, an approach to PrEP scale up that focuses solely on HIV risk reduction without consideration of individuals' mental health and psychosocial needs is untenable. ${ }^{4}$

In this analysis, we describe the burden of mental disorders among populations that are likely to benefit from PrEP. We review findings showing that mental disorders may undermine long-term persistence with PrEP and therefore warrant the attention of researchers and implementers seeking to maximise the effectiveness of PrEP at the population level. We conclude that the ambitious scale up of PrEP offers an opportunity to undertake concurrent scale up of screening and treatment for mental disorders, both as a strategy to improve PrEP effectiveness and as a pathway to expand access to mental healthcare among populations at elevated risk of HIV acquisition.

\section{MENTAL DISORDERS AMONG PREP USERS: A THREAT TO EFFECTIVE IMPLEMENTATION}

Mental disorders are highly prevalent among populations likely to benefit from PrEP. Prevalence data are most robust for sexual-minority and gender-minority populations in high-income settings, where population-based surveys have reported rates of depression, anxiety and other mental disorders that consistently exceed those in the general population by a factor of two. ${ }^{14}$ Because many key populations are marginalised, reaching them has been a challenge for researchers in LMICs. Other populations, such as adolescent girls and young women, may not be resourced or empowered to engage in sexual health. ${ }^{15}$ Data from LMICs and among key populations such as sex workers are often not routinely collected, but available studies have demonstrated mental health disparities of an equivalent-if not greater-magnitude compared with those observed in high-income settings. ${ }^{16}$ Studies of PrEP users, in particular, document high rates of mental disorders and poor mental health. Table 1 presents studies identified as part of a scoping review of electronic databases (ie, EMBASE, PubMed, Web of Science, PsycINFO) to examine the prevalence of mental disorders among PrEP candidates.

The prevalence of mental disorders in these populations has been associated with differential exposure to numerous structural causes, including stigma, ${ }^{17}$ racism $^{18}$ and interpersonal violence. ${ }^{19}$ In particular, stigma is highly prevalent among these populations, and has been labelled a 'fundamental cause' of health inequalities. ${ }^{20}$ Stigma is defined as 'labelling, stereotyping, separation, status loss and discrimination in a context in which power is exercised, ${ }^{20}$ and may be associated with multiple, simultaneously held statuses and identities, including race, gender expression, sexual orientation and presumed HIV status. Stigma has been linked to mental disorders through increased social isolation, maladaptive coping, and stress. ${ }^{21-23}$ Moreover, stigma frequently interacts with other structural causes, such as racism and interpersonal violence, to further worsen mental health. Although stigma remains a worthwhile target of public health intervention, attention to its downstream consequences, such as poor mental health, is also warranted.

Across all settings, mental disorders remain underdiagnosed and undertreated, contributing to significant morbidity. ${ }^{24}$ Mental disorders have also been linked to poorer physical health, in part due to their association with self-harm, substance use disorders and disability, but also due to reduced care seeking and limited engagement in longitudinal care. While evidence of the association between mental disorders and physical health is strongest for chronic conditions that typically require intensive self-management such as diabetes, heart failure and HIV, emerging data suggest that mental disorders may also undermine PrEP persistence. For example, a study by Krakower et al of 663 people who initiated PrEP through a Boston community health centre specialising in healthcare for sexual and gender minorities found that those with a mental health diagnosis were 1.2 times more likely to discontinue PrEP compared with those without a mental health diagnosis. ${ }^{9}$ The study also showed a compounding risk of discontinuation with increasing burden of mental illness, a concerning finding given the phenomenon of syndemic disease burden within some key populations. ${ }^{11}{ }^{25-27}$ A study of PrEP service users in Wales (UK) similarly found a $29 \%$ prevalence of mental health problems among people who were either poorly engaged in PrEP care or who were lost to follow-up. ${ }^{28}$ Qualitative studies conducted in high-income countries $^{2930}$ and low-income countries ${ }^{31}$ have also implicated 


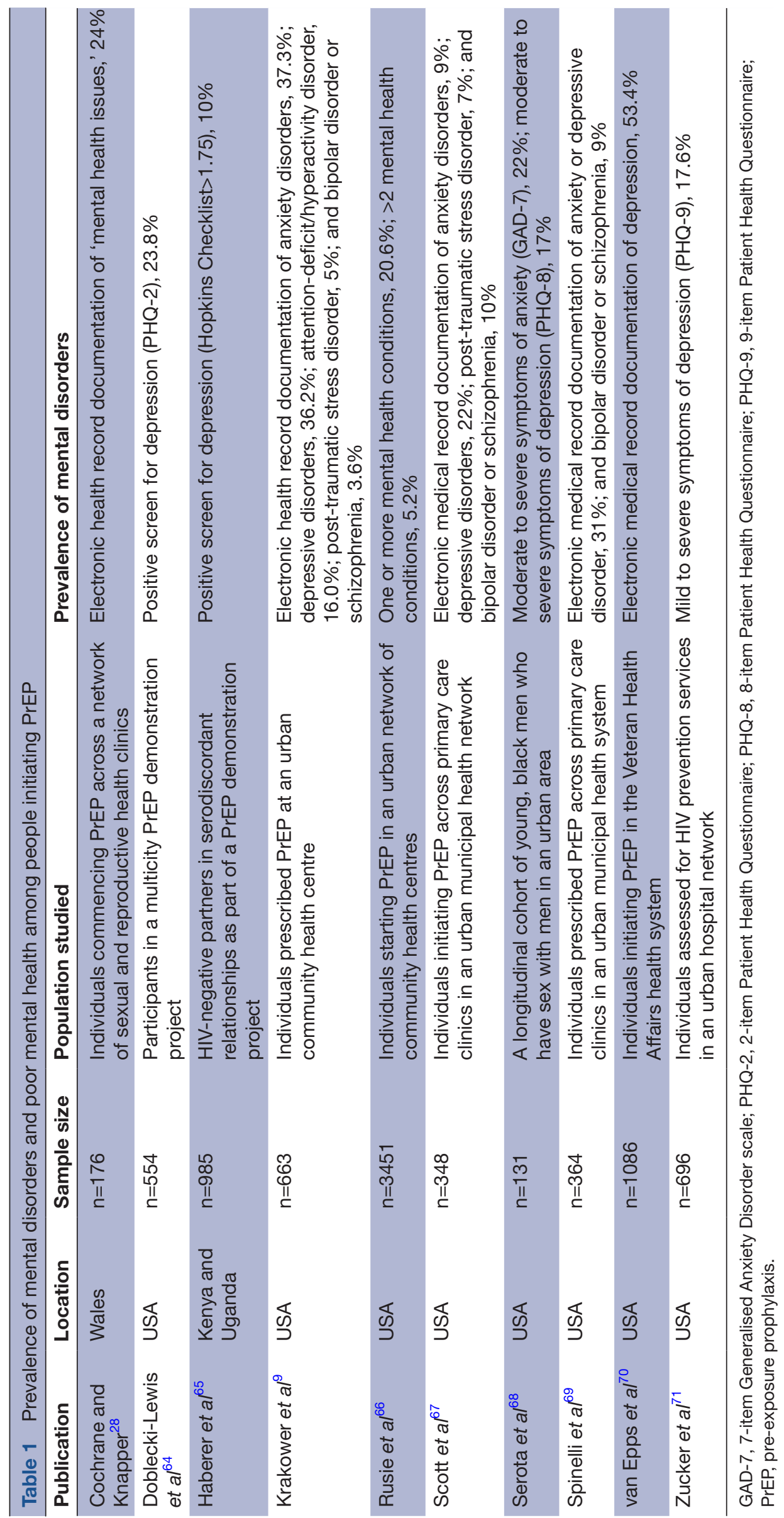


substance use and mental health problems in PrEP nonadherence and/or discontinuation.

Despite the observed association between mental disorders and PrEP discontinuation, current studies are limited by the lack of consensus about how PrEP discontinuation is defined, the poorly understood reasons for why people discontinue PrEP, and whether discontinuation coincides with potential HIV exposure. ${ }^{32}$ There are also few studies from LMICs, and few studies of mental health and PrEP engagement in certain populations such as at-risk cisgender women. ${ }^{33}$ Despite these shortcomings, the high burden of mental disorders among first-time initiators of PrEP is cause for concern, and represents a potential roadblock in efforts to maximise PrEP's longterm effectiveness at the population level.

\section{PREP AS A GATEWAY TO ENGAGEMENT IN LONGITUDINAL HEALTHCARE AND COMMUNITY MENTAL HEALTH}

International guidelines recommend that PrEP users attend clinical visits every 3 months to screen for HIV seroconversion, address barriers to prevention-effective adherence and provide ongoing sexual health services. ${ }^{8}$ These guidelines have led some researchers to question whether quarterly visits are too burdensome for patients, and have spurred the development of alternative models. ${ }^{34}$ These ongoing discussions notwithstanding, the movement of HIV prevention into the clinic presents a unique opportunity to embed PrEP within primary care, which could engage key populations in care for chronic health and mental health conditions.

PrEP may serve as a gateway to primary care and preventive services. In a cohort of PrEP-eligible individuals in the USA, investigators found that those subsequently prescribed PrEP were also more likely to receive preventive health screening, including influenza vaccination, tobacco screening, glucose testing and depression screening. ${ }^{35}$ A similar phenomenon has been reported in LMIC settings, ${ }^{36}$ where recent work from South Africa has documented a so-called 'antiretroviral therapy (ART) advantage. ${ }^{37}$ As part of this 'advantage,' this work has shown that individuals engaged in ART programmes are more likely to receive screening for hypertension and diabetes, and are also more likely to achieve adequate control of these conditions relative to others in the general population. ${ }^{37}$

Findings from these studies bolster a consensus that integrated care delivery is both feasible and necessary to support the health needs of populations affected by HIV across the life course. ${ }^{38}$ Unfortunately, limited attention is being paid to mental disorders by implementers within these integration efforts, particularly in LMICs, where efforts to expand access to mental healthcare have focused primarily on small-scale efficacy and effectiveness studies rather than implementation strategies for population-wide scale up. ${ }^{39}$ Moreover, with HIV in particular, efforts to integrate mental healthcare into primary care settings have largely occurred in the context of treatment programmes, with little consideration of how prevention programmes-and HIV testing programmes-might benefit from these efforts. ${ }^{40}$ Across all settings, the high burden of mental disorders in key populations-coupled with findings that PrEP initiation can spur engagement in primary healthcare-points to the high likelihood that offering routine screening and treatment for mental disorders as part of HIV prevention programming will yield benefits for both HIV prevention and mental health.

\section{PREP AND MENTAL HEALTHCARE: PROMISES OF BENEFIT}

As the global scale up of PrEP continues, there is a growing appreciation of the many barriers that influence an individual's choice to initiate and remain engaged with PrEP. Although PrEP only reduces the risk of HIV acquisition, encounters with the health system for PrEP initiation offer an ideal opportunity to address a number of these other barriers, including undiagnosed or undertreated mental illness, that can undermine long-term engagement in HIV prevention behaviours. PrEP use may also be beneficial for emotional well-being through empowerment and reduced anxiety about contracting HIV, ${ }^{41}$ further underscoring the potential benefits to be gained from addressing mental disorders and HIV prevention as part of an integrated approach to care delivery.

Although the goals of care integration are the same across contexts, what integration looks like in practice may vary, and will require programmes to implement models that can be embedded within the local health system. Possible models include incorporating screening for mental disorders within PrEP programming, situating HIV risk screening within mental healthcare, or embedding both PrEP and mental health screening within primary care. ${ }^{42}$ Regardless of the model that is selected, we believe that any implementation effort should be driven by three principles that affirm the importance of patient choice and enable delivery of care that is sensitive to the context-specific barriers individuals may face in accessing PrEP.

1. Low-threshold care: While PrEP initiation is an opportunity for engagement in longitudinal care, access to PrEP should be as low-threshold as possible. ${ }^{43}$ Screening and subsequent treatment for mental disorders at PrEP initiation may improve the long-term effectiveness of PrEP but should not be used as a condition for initiating PrEP. Ideally, screening for common mental disorders could occur during initial prescription, with periodic reassessment and an offer of treatment initiation or referral if clinically indicated. Collaborative care or stepped-care approaches may serve as useful models in this regard. ${ }^{44-46}$ Instruments used to screen for common mental disorders have been validated across diverse contexts, take only minutes to administer, and can be readily adapted to populations with low levels of literacy. ${ }^{47}$ Yet, each additional instrument poses a real burden in the workflow of a 
healthcare worker. Wherever possible, efforts to minimise the burdens of PrEP care on patients, providers, and health systems should be pursued. ${ }^{48}$ Ultrashort screening instruments are also available. ${ }^{4-51}$ Finally, strategies such as mHealth solutions, provider-facing alerts and task sharing may be helpful in streamlining inclusion of mental health screening as part of this process.

2. Access to mental healthcare: Screening in the absence of effective treatment raises important ethical and logistical concerns. ${ }^{52}$ Non-pharmacological approaches to mental health treatment-cognitive-behavioural therapy, mindfulness, stress management, peer counselling and motivational interviewinghave been studied among people living with HIV across multiple contexts. ${ }^{53}$ These may be of particular importance in LMICs where there is limited access to pharmacotherapies, although due to chronic underfunding the capacity of many LMIC mental health systems to deliver non-pharmacological treatments is also limited ${ }^{54}$ However, questions of how these interventions ought to be delivered in the context of PrEP care remain largely unanswered, including which cadres of healthcare workers can participate in task sharing, how care might be escalated in patients with severe or worsening mental disorders, and whether medication management or abbreviated interventions might feasibly be completed as part of quarterly PrEP visits in primary care. Importantly, answers to these questions may vary according to geography, cultural context, regulatory context, target population, and other factors. ${ }^{55}$ New PrEP agents will raise new questions about delivery. ${ }^{56}$ Addressing these and related questions will be crucial to the success of integration efforts and would benefit from a rigorous implementation science approach that considers how evidence-based treatment interventions might be adapted to local contexts. ${ }^{57}$

3. Stigma reduction: Stigma is one of the most potent barriers to accessing PrEP ${ }^{58}$ mental healthcare ${ }^{59}$ and substance use treatment ${ }^{60}$ and may imperil integration efforts if not adequately addressed. Individuals with mental disorders and an indication for PrEP face multiple, potentially layered stigmas including those associated with HIV, mental illness and identification with marginalised groups such as sex workers and men who have sex with men. ${ }^{61}$ These stigmas often reinforce one another, and can be abetted by healthcare settings that are unsupportive or unprepared to respond to individuals' unique care needs. ${ }^{62}$ Evidence-based approaches for addressing health-related stigma in healthcare settings exist ${ }^{62}{ }^{63}$ but are often underused in practice. As integration of PrEP and mental healthcare is considered, stigma-reduction efforts will be crucial to ensuring that patients' encounters with the health system lead to long-term engagement rather than to further distancing from needed care.

\section{CONCLUSION}

If successful, the global roll-out of PrEP has the potential to transform how millions of at-risk individuals achieve HIV prevention. To maximise PrEP's effectiveness, however, attention must be paid to the cascade of PrEP care beyond awareness, acceptability, and dispensing, in order to ensure that PrEP users experience adequate and persisting protection from HIV acquisition. As HIV prevention programmes seek to secure the engagement of key populations in the long term, integration of PrEP and mental healthcare offers an opportunity both to address the high burden of mental disorders in these populations and to maximise PrEP adherence and persistence. Implementation efforts must be low threshold, recognise differential access to mental healthcare, and emphasise stigma reduction. However, limitations will include overburdened human resources of healthcare; geographical, cultural and systems-related barriers to implementation; and unrecognised and unaddressed dimensions of stigma.

Contributors DJl conceived the initial idea for the manuscript and wrote the first draft. All authors provided critical feedback and approved the final submission.

Funding The authors have not declared a specific grant for this research from any funding agency in the public, commercial or not-for-profit sectors.

Competing interests JEH is a consultant for Merck. ACT receives a stipend from Elsevier for his work as co-editor in chief of Social Science and Medicine-Mental Health.

Patient consent for publication Not applicable.

Ethics approval This study does not involve human participants.

Provenance and peer review Not commissioned; externally peer reviewed.

\section{Data availability statement Not applicable.}

Open access This is an open access article distributed in accordance with the Creative Commons Attribution Non Commercial (CC BY-NC 4.0) license, which permits others to distribute, remix, adapt, build upon this work non-commercially, and license their derivative works on different terms, provided the original work is properly cited, appropriate credit is given, any changes made indicated, and the use is non-commercial. See: http://creativecommons.org/licenses/by-nc/4.0/.

\section{ORCID iDs}

Daniel J Ikeda http://orcid.org/0000-0001-7338-0778

Alexander C Tsai http://orcid.org/0000-0001-6397-7917

\section{REFERENCES}

1 UNAIDS. Confronting inequalities: Lessons for pandemic responses from 40 years of AIDS [Internet], 2021. Available: https://www. unaids.org/sites/default/files/media_asset/2021-global-aids-update_ en.pdf

2 Grant RM, Lama JR, Anderson PL, et al. Preexposure chemoprophylaxis for HIV prevention in men who have sex with men. N Engl J Med 2010;363:2587-99.

3 Molina J-M, Capitant C, Spire B, et al. On-Demand preexposure prophylaxis in men at high risk for HIV-1 infection. N Engl J Med 2015;373:2237-46.

4 Rivet Amico K, Bekker L-G. Global PrEP roll-out: recommendations for programmatic success. Lancet HIV 2019;6:e137-40.

5 Nunn AS, Brinkley-Rubinstein L, Oldenburg CE, et al. Defining the HIV pre-exposure prophylaxis care continuum. AIDS 2017;31:731-4.

6 Spinelli MA, Buchbinder SP. Pre-exposure prophylaxis persistence is a critical issue in PrEP implementation. Clin Infect Dis 2020;71:583-5.

7 Haberer JE, Bangsberg DR, Baeten JM, et al. Defining success with HIV pre-exposure prophylaxis: a prevention-effective adherence paradigm. AIDS 2015;29:1277-85. 
8 Rutstein SE, Smith DK, Dalal S, et al. Initiation, discontinuation, and restarting HIV pre-exposure prophylaxis: ongoing implementation strategies. Lancet HIV 2020;7:e721-e730.

9 Krakower D, Maloney KM, Powell VE, et al. Patterns and clinical consequences of discontinuing HIV preexposure prophylaxis during primary care. J Int AIDS Soc 2019;22:e25250.

10 Spinelli MA, Laborde N, Kinley P, et al. Missed opportunities to prevent HIV infections among pre-exposure prophylaxis users: a population-based mixed methods study, San Francisco, United States. J Int AIDS Soc 2020;23:e25472.

11 Stall R, Mills TC, Williamson J, et al. Association of co-occurring psychosocial health problems and increased vulnerability to HIV/ AIDS among urban men who have sex with men. Am J Public Health 2003;93:939-42.

12 Safren SA, Reisner SL, Herrick A, et al. Mental health and HIV risk in men who have sex with men. J Acquir Immune Defic Syndr 2010;55(Suppl 2):S74-7.

13 Celum CL, Delany-Moretlwe S, Baeten JM, et al. HIV pre-exposure prophylaxis for adolescent girls and young women in Africa: from efficacy trials to delivery. J Int AIDS Soc 2019;22(Suppl 4):e25298.

14 Gonzales G, Henning-Smith C. Health disparities by sexual orientation: results and implications from the behavioral risk factor surveillance system. J Community Health 2017;42:1163-72.

15 de Dieu Tapsoba J, Zangeneh SZ, Appelmans E. Persistence of oral pre-exposure prophylaxis (PrEP) among adolescent girls and young women initiating PrEP for HIV prevention in Kenya. AIDS Care 2020:1-9.

16 Beattie TS, Smilenova B, Krishnaratne S, et al. Mental health problems among female sex workers in low- and middle-income countries: a systematic review and meta-analysis. PLoS Med 2020;17:e1003297.

17 Meyer IH. Prejudice, social stress, and mental health in lesbian, gay, and bisexual populations: conceptual issues and research evidence. Psychol Bull 2003;129:674-97.

18 Bogart LM, Wagner GJ, Galvan FH, et al. Perceived discrimination and mental health symptoms among black men with HIV. Cultur Divers Ethnic Minor Psychol 2011;17:295-302.

19 Deering KN, Amin A, Shoveller J, et al. A systematic review of the correlates of violence against sex workers. Am J Public Health 2014;104:e42-54.

20 Hatzenbuehler ML, Phelan JC, Link BG. Stigma as a fundamental cause of population health inequalities. Am J Public Health 2013;103:813-21.

21 Link BG, Cullen FT, Struening E, et al. A modified labeling theory approach to mental disorders: an empirical assessment. Am Sociol Rev 1989;54:400.

22 Tsai AC, Bangsberg DR, Frongillo EA, et al. Food insecurity, depression and the modifying role of social support among people living with HIV/AIDS in rural Uganda. Soc Sci Med 2012;74:2012-9.

23 Takada S, Weiser SD, Kumbakumba E, et al. The dynamic relationship between social support and HIV-related stigma in rural Uganda. Ann Behav Med 2014;48:26-37.

24 Thornicroft G, Chatterji S, Evans-Lacko S, et al. Undertreatment of people with major depressive disorder in 21 countries. Br J Psychiatry 2017;210:119-24.

25 Mustanski B, Andrews R, Herrick A, et al. A syndemic of psychosocial health disparities and associations with risk for attempting suicide among young sexual minority men. Am J Public Health 2014;104:287-94.

26 Tsai AC, Burns BFO. Syndemics of psychosocial problems and HIV risk: a systematic review of empirical tests of the disease interaction concept. Soc Sci Med 2015;139:26-35.

27 Tsai AC, Mendenhall E, Trostle JA, et al. Co-occurring epidemics, syndemics, and population health. Lancet 2017;389:978-82.

28 Cochrane E, Knapper C. Audit of patient retention in pre-exposure prophylaxis (PrEP) services in an integrated sexual reproductive health service setting. Int J STD AIDS 2019;30:1432-5.

29 Edeza A, Karina Santamaria E, Valente PK, et al. Experienced barriers to adherence to pre-exposure prophylaxis for HIV prevention among MSM: a systematic review and meta-ethnography of qualitative studies. AIDS Care 2021;33:1-9.

30 Nydegger LA, Dickson-Gomez J, Ko TK. Structural and syndemic barriers to PrEP adoption among black women at high risk for HIV: a qualitative exploration. Cult Health Sex 2021;23:659-73.

31 Van der Elst EM, Mbogua J, Operario D, et al. High acceptability of HIV pre-exposure prophylaxis but challenges in adherence and use: qualitative insights from a phase I trial of intermittent and daily PrEP in at-risk populations in Kenya. AIDS Behav 2013;17:2162-72.

32 Stankevitz K, Grant H, Lloyd J. Oral pre-exposure prophylaxis (PrEP) continuation, measurement, and reporting: a systematic review and meta-analysis. AIDS 2020.
33 Hirschhorn LR, Brown RN, Friedman EE, et al. Black Cisgender women's PrEP knowledge, attitudes, preferences, and experience in Chicago. J Acquir Immune Defic Syndr 2020;84:497-507.

34 Siegler AJ, Mayer KH, Liu AY, et al. Developing and assessing the feasibility of a home-based preexposure prophylaxis monitoring and support program. Clin Infect Dis 2019;68:501-4.

35 Marcus JL, Levine K, Grasso C, et al. HIV preexposure prophylaxis as a gateway to primary care. Am J Public Health 2018;108:1418-20.

36 Mitton JA, North CM, Muyanja D, et al. Smoking cessation after engagement in HIV care in rural Uganda. AIDS Care 2018;30:1622-9.

37 Manne-Goehler J, Montana L, Gómez-Olivé FX, et al. The ART advantage: health care utilization for diabetes and hypertension in rural South Africa. J Acquir Immune Defic Syndr 2017;75:561-7.

38 Safreed-Harmon K, Anderson J, Azzopardi-Muscat N, et al. Reorienting health systems to care for people with HIV beyond viral suppression. Lancet HIV 2019;6:e869-77.

39 Wagenaar BH, Hammett WH, Jackson C, et al. Implementation outcomes and strategies for depression interventions in low- and middle-income countries: a systematic review. Glob Ment Health 2020;7:e7.

40 Remien RH, Stirratt MJ, Nguyen N, et al. Mental health and HIV/ AIDS: the need for an integrated response. AIDS 2019;33:1411-20.

41 Grant RM, Koester KA. What people want from sex and preexposure prophylaxis. Curr Opin HIV AIDS 2016;11:3-9.

42 Duffy M, Ojikutu B, Andrian S, et al. Non-communicable diseases and HIV care and treatment: models of integrated service delivery. Trop Med Int Health 2017;22:926-37.

43 Laborde ND, Kinley PM, Spinelli M, et al. Understanding PrEP persistence: provider and patient perspectives. AIDS Behav 2020;24:2509-19.

44 Pyne JM, Fortney JC, Curran GM, et al. Effectiveness of collaborative care for depression in human immunodeficiency virus clinics. Arch Intern Med 2011;171:23-31.

45 Tsai AC, Morton SC, Mangione CM, et al. A meta-analysis of interventions to improve care for chronic illnesses. Am J Manag Care 2005:11:478-88.

46 Unützer J, Katon W, Callahan CM, et al. Collaborative care management of late-life depression in the primary care setting: a randomized controlled trial. JAMA 2002;288:2836-45.

47 Ali G-C, Ryan G, De Silva MJ. Validated screening tools for common mental disorders in low and middle income countries: a systematic review. PLoS One 2016;11:e0156939.

48 Siegler AJ, Steehler K, Sales JM, et al. A review of HIV preexposure prophylaxis streamlining strategies. Curr HIVIAIDS Rep 2020;17:643-53.

49 Rochat TJ, Tomlinson M, Newell M-L, et al. Detection of antenatal depression in rural HIV-affected populations with short and ultrashort versions of the Edinburgh postnatal depression scale (EPDS). Arch Womens Ment Health 2013;16:401-10.

50 Hung KJ, Tomlinson M, le Roux IM, et al. Community-based prenatal screening for postpartum depression in a South African township. Int $J$ Gynaecol Obstet 2014;126:74-7.

51 Tsai AC, Tomlinson M, Dewing S, et al. Antenatal depression case finding by community health workers in South Africa: feasibility of a mobile phone application. Arch Womens Ment Health 2014;17:423-31.

52 Kagee A, Tsai AC, Lund C, et al. Screening for common mental disorders in low resource settings: reasons for caution and a way forward. Int Health 2013;5:11-14.

53 Sikkema KJ, Dennis AC, Watt MH, et al. Improving mental health among people living with HIV: a review of intervention trials in low- and middle-income countries. Glob Ment Health 2015;2. doi:10.1017/gmh.2015.17. [Epub ahead of print: 0909 2015]

54 Jacob KS, Sharan P, Mirza I, et al. Mental health systems in countries: where are we now? Lancet 2007;370:1061-77.

55 Underhill K, Operario D, Mimiaga MJ, et al. Implementation science of pre-exposure prophylaxis: preparing for public use. Curr HIVIAIDS Rep 2010;7:210-9.

56 Coelho LE, Torres TS, Veloso VG, et al. Pre-exposure prophylaxis 2.0: new drugs and technologies in the pipeline. Lancet HIV 2019:6:e788-99.

57 Betancourt TS, Chambers DA. Optimizing an era of global mental health implementation science. JAMA Psychiatry 2016;73:99-100.

58 Golub SA. Prep stigma: implicit and explicit drivers of disparity. Curr HIVIAIDS Rep 2018;15:190-7.

59 Clement S, Schauman O, Graham T, et al. What is the impact of mental health-related stigma on help-seeking? A systematic review of quantitative and qualitative studies. Psychol Med 2015;45:11-27. 
60 Tsai AC, Kiang MV, Barnett ML, et al. Stigma as a fundamental hindrance to the United States opioid overdose crisis response. PLoS Med 2019;16:e1002969.

61 Herek GM. Aids and stigma. Am Behav Sci 1999:42:1106-16.

62 Nyblade L, Stockton MA, Giger K, et al. Stigma in health facilities: why it matters and how we can change it. BMC Med 2019;17:25.

63 Ikeda DJ, Nyblade L, Srithanaviboonchai K, et al. A quality improvement approach to the reduction of HIV-related stigma and discrimination in healthcare settings. BMJ Glob Health 2019;4:e001587.

64 Doblecki-Lewis S, Liu AY, Feaster DJ, et al. Patterns and correlates of participant retention in a Multi-City pre-exposure prophylaxis demonstration project. J Acquir Immune Defic Syndr 2018;79:62-9.

65 Haberer JE, Kidoguchi L, Heffron R, et al. Alignment of adherence and risk for HIV acquisition in a demonstration project of preexposure prophylaxis among HIV serodiscordant couples in Kenya and Uganda: a prospective analysis of prevention-effective adherence. J Int AIDS Soc 2017;20:21842.

66 Rusie LK, Orengo C, Burrell D, et al. Preexposure prophylaxis initiation and retention in care over 5 years, 2012-2017: are Quarterly visits too much? Clin Infect Dis 2018;67:283-7.
67 Scott HM, Spinelli M, Vittinghoff E, et al. Racial/ethnic and HIV risk category disparities in preexposure prophylaxis discontinuation among patients in publicly funded primary care clinics. AIDS 2019;33:2189-95.

68 Serota DP, Rosenberg ES, Sullivan PS, et al. Pre-exposure prophylaxis uptake and discontinuation among young black men who have sex with men in Atlanta, Georgia: a prospective cohort study. Clin Infect Dis 2020;71:574-82.

69 Spinelli MA, Scott HM, Vittinghoff E, et al. Missed visits associated with future preexposure prophylaxis (PrEP) discontinuation among PreP users in a municipal primary care health network. Open Forum Infect Dis 2019;6:ofz101.

70 van Epps P, Maier M, Lund B, et al. Medication adherence in a nationwide cohort of Veterans initiating pre-exposure prophylaxis (PrEP) to prevent HIV infection. J Acquir Immune Defic Syndr 2018;77:272-8.

71 Zucker J, Carnevale C, Richards P, et al. Predictors of disengagement in care for individuals receiving pre-exposure prophylaxis (PrEP). J Acquir Immune Defic Syndr 2019;81:e104-8. 